ANNALES

POLONICI MATHEMATICI

LXXVII.1 (2001)

\title{
On the solvability of the Lyapunov equation for nonselfadjoint differential operators of order $2 m$ with nonlocal boundary conditions
}

\author{
by ARIs TERSENOv (Heraklion)
}

\begin{abstract}
This paper is devoted to the solvability of the Lyapunov equation $A^{*} U+$ $U A=I$, where $A$ is a given nonselfadjoint differential operator of order $2 m$ with nonlocal boundary conditions, $A^{*}$ is its adjoint, $I$ is the identity operator and $U$ is the selfadjoint operator to be found. We assume that the spectra of $A^{*}$ and $-A$ are disjoint. Under this restriction we prove the existence and uniqueness of the solution of the Lyapunov equation in the class of bounded operators.
\end{abstract}

1. Introduction. Consider the Cauchy problem in a Hilbert space $H$ :

$$
\frac{d u}{d t}=\mathbf{A} u(t), \quad t>0 ; \quad u(0)=u_{0},
$$

where $\mathbf{A}$ is a closed linear operator with domain $D(\mathbf{A})$ dense in $H$. It is assumed that this problem is abstract parabolic, i.e. for any initial data $u_{0} \in H$ there is a continuous function $u(t)$, which is defined for $t>0$, takes values in $D(\mathbf{A})$ and satisfies (1.1). Many initial boundary value problems for evolution equations reduce to problem (1.1). Assume that $\mathbf{A}$ is a nonselfadjoint differential operator of order $2 m$, satisfying the following condition, which ensures the abstract parabolicity of problem (1.1) (see [10]):

$1^{\circ}$ For some real $\beta$ and $\theta(\pi / 2<\theta<\pi)$, the resolvent $R(\mathbf{A}, \lambda)=$ $(\mathbf{A}-\lambda I)^{-1}$ of $\mathbf{A}$ exists and the quantity $\|\lambda R(\mathbf{A}, \lambda)\|$ is uniformly bounded with respect to $\lambda$ in the sector $|\arg (\lambda-\beta)| \leq \theta$.

Suppose that the operator $\mathbf{A}$ has a discrete spectrum. It is known that the stability of solutions of problem (1.1) depends on the location of the spectrum of $\mathbf{A}$. The sum of the algebraic multiplicities of the eigenvalues

2000 Mathematics Subject Classification: 34G10, 34B05, 47E05, 47N20.

Key words and phrases: Lyapunov equation, compatibility conditions, nonlocal boundary conditions.

Partially supported by Russian Foundation for Basic Research (code 97-01-00508). 
of $\mathbf{A}$ in the half-plane $\Re(\lambda) \geq 0$ minus the number of linearly independent eigenelements corresponding to the imaginary eigenvalues is called the instability index of $\mathbf{A}$. A direct computation of the spectrum is a laborious work, therefore for applications it is important to find effective criteria for counting instability indices. This problem is completely solved for selfadjoint ordinary differential equations. Using the methods of variational calculus Morse [11], [12] reduced the problem of counting the instability indices to the problem of calculating the number of conjugate points of some boundary value problem. Similar results were obtained by Krein [6], who used a different approach. The theory of instability indices started in the works of Morse and Krein and was completed by Zelenyak [4] and Belov [5].

Extending these results to nonselfadjoint operators is complicated due to difficulties in the use of the methods of variational calculus. A constructive approach to solving this problem for matrices was suggested by Lyapunov, who reduced the problem of investigating the stability of a nonselfadjoint matrix $\mathbf{A}$ to the problem of investigating the stability of some selfadjoint matrix $\mathbf{U}$. To construct such a $\mathbf{U}$ one has to solve an equation of the following type:

$$
\mathbf{A}^{*} \mathbf{U}+\mathbf{U A}=\mathbf{V}
$$

where $\mathbf{A}$ is the given matrix, $\mathbf{A}^{*}$ is its adjoint, $\mathbf{V}=\mathbf{V}^{*}, \mathbf{V}>0, \mathbf{U}$ is the selfadjoint matrix to be found. Lyapunov showed that if $\mathbf{A}$ is stable, then so is $\mathbf{U}$ and vice versa. Krein [6] generalized these results to bounded operators in infinite-dimensional spaces. Belonosov [1] extended them to the case of unbounded operators. He proved that if $\mathbf{A}$ satisfies $1^{\circ}$ as well as the conditions:

$2^{\circ}$ The spectra of $\mathbf{A}^{*}$ and $-\mathbf{A}$ are disjoint,

$3^{\circ}$ The operator $\mathbf{V}$ in (1.2) is bounded,

then (1.2) has no more than one solution in the class of bounded operators and the instability indices of $\mathbf{A}$ and $\mathbf{U}$ coincide.

The question of the properties of the solution of the Lyapunov equation (1.2) when $\mathbf{A}$ is a nonselfadjoint differential operator of order $2 m$ with local boundary conditions in spaces of vector-valued functions with one independent variable was investigated in [1]-[3]. The present work is concerned with equation (1.2) when $\mathbf{A}$ is a nonselfadjoint operator of order $2 m$ with nonlocal boundary conditions in spaces of vector-valued functions with one independent variable. We assume that conditions $1^{\circ}-3^{\circ}$ are satisfied. As $\mathbf{V}$ we take the identity operator in $L_{2}$. We look for a solution of (1.2) in integral form. The kernel of this integral satisfies certain conditions which will be explained in what follows. After substitution of this integral operator into (1.2) we obtain an elliptic boundary value problem in a square domain with 
respect to the kernel of the integral operator. This kernel also satisfies an additional condition: the normal derivative of the kernel has a discontinuity on the diagonal of the square. We construct a solution of the boundary value problem mentioned above. As a final result we prove the existence and uniqueness of the solution of the Lyapunov equation in the class of bounded operators. The results of this work can be used in the investigation of instability indices of nonselfadjoint operators of order $2 m$ with nonlocal boundary conditions.

2. Boundary value problem. Denote by $\mathbb{C}$ the set of complex numbers and by $\mathbb{C}_{n}$ the $n$-dimensional complex space. A vector $u \in \mathbb{C}_{n}$ is regarded as a column with components $u^{1}, \ldots, u^{n}$. The set of complex matrices $B=\left(b^{i j}\right), 1 \leq i \leq n, 1 \leq j \leq m$, is denoted by $\mathbb{C}_{n \times m}$, and $B^{*} \in \mathbb{C}_{m \times n}$ is the matrix adjoint to $B$. We use the standard spaces $L_{2}(\Omega, G), W_{2}^{k}(\Omega, G)$, $C^{k}(\bar{\Omega}, G)$ of functions defined on $\Omega$ or $\bar{\Omega}$ and taking values in $G$, where $\Omega \subset \mathbb{R}_{m}$, and $G$ is either $\mathbb{C}_{n}$ or $\mathbb{C}_{n \times n}$. Notice that $L_{2}\left(\Omega, \mathbb{C}_{n}\right)$ is a Hilbert space with inner product

$$
(u, v)=\int_{\Omega} v^{*}(x) u(x) d x .
$$

Consider the nonselfadjoint operator $\mathbf{A}$ on the interval $\omega=\{0<x<1\}$, generated by the differential expression

$$
l(u)=u^{(2 m)}(x)+P_{1}(x) u^{(2 m-1)}(x)+\ldots+P_{2 m}(x) u(x)
$$

and the regular boundary conditions [13]

$$
\begin{aligned}
B_{i}^{1} u^{\left(k_{i}\right)}(0)+ & \sum_{j=0}^{k_{i}-1} B_{i j}^{1} u^{(j)}(0)+B_{i}^{2} u^{\left(k_{i}\right)}(1)+\sum_{j=0}^{k_{i}-1} B_{i j}^{2} u^{(j)}(1)=0, \\
& \quad i=1, \ldots, 2 m, 0 \leq k_{2 m} \leq \ldots \leq k_{1} \leq 2 m-1, k_{i+2}<k_{i},
\end{aligned}
$$

where at least one of the matrices $B_{i}^{1}, B_{i}^{2}$ is not zero. The coefficients $P_{k}(x)$ belong to $C^{2 m-k+\varepsilon}\left([0,1], \mathbb{C}_{n \times n}\right)$, where $0<\varepsilon<1$. The domain $D(\mathbf{A})$ consists of the functions $u \in W_{2}^{2 m}\left(\omega, \mathbb{C}_{n}\right)$ which satisfy the given boundary conditions. It is known [13] that $\mathbf{A}$ acts in $L_{2}\left(\omega, \mathbb{C}_{n}\right)$, is closed, has a discrete spectrum and satisfies $1^{\circ}$. We assume that $\mathbf{A}$ satisfies $2^{\circ}$ and, without loss of generality, that for any $|\lambda|$ the following estimate holds:

$$
\left\|(\mathbf{A}-\lambda I)^{-1}\right\| \leq \frac{c}{1+|\lambda|}, \quad|\arg (\lambda-\beta)| \leq \theta .
$$

The resolvent of $\mathbf{A}$ is an integral operator with continuous kernel, acting in $L_{2}\left(\omega, \mathbb{C}_{n}\right)$. The adjoint operator $\mathbf{A}^{*}$ is generated by the differential expression $[13]$

$$
m(v)=v^{(2 m)}(x)+(-1)^{2 m-1}\left(P_{1}^{*}(x) v(x)\right)^{(2 m-1)}+\ldots+P_{2 m}^{*} v(x)
$$


and the regular boundary conditions of the same type, but with other coefficients:

$$
\widetilde{B}_{i}^{1} v^{\left(k_{i}\right)}(0)+\sum_{j=0}^{k_{i}-1} \widetilde{B}_{i j}^{1} v^{(j)}(0)+\widetilde{B}_{i}^{2} v^{\left(k_{i}\right)}(1)+\sum_{j=0}^{k_{i}-1} \widetilde{B}_{i j}^{2} v^{(j)}(1)=0 .
$$

The operator $\mathbf{A}^{*}$ also acts in $L_{2}\left(\omega, \mathbb{C}_{n}\right)$ and its resolvent is an integral operator with continuous kernel. We say that the boundary condition is of order $k_{i}$ if it does not involve $u^{(\nu)}(p), p=0,1$, when $\nu>k_{i}$ and at least one of the matrices $B_{i}^{1}, B_{i}^{2}$ is not zero.

Consider equation (1.2) with coefficients $\mathbf{A}$ and $\mathbf{A}^{*}$. Let $\mathbf{V}=\mathbf{I}$, where $\mathbf{I}$ is the identity operator. We look for a solution of equation (1.2) in integral form:

$$
\mathbf{U} u(x)=\int_{0}^{1} U(x, y) u(y) d y,
$$

where the kernel $U(x, y)$ is defined in the domain $\Omega=\{(x, y): 0<x<1$, $0<y<1\}$, takes values in $\mathbb{C}_{n \times n}$ and satisfies the following conditions:

1. $U \in W_{2}^{2 m-1}\left(\Omega, \mathbb{C}_{n \times n}\right)$.

2. $U_{ \pm} \in W_{2}^{2 m}\left(\Omega_{ \pm}^{\delta}, \mathbb{C}_{n \times n}\right)$ for any $\delta>0$, where

$$
\begin{aligned}
& \Omega_{+}^{\delta}=\{(x, y) \in \Omega: y<x, x-y<1-\delta, \delta<(x+y) / 2<1-\delta\}, \\
& \Omega_{-}^{\delta}=\{(x, y) \in \Omega: x<y, y-x<1-\delta, \delta<(x+y) / 2<1-\delta\},
\end{aligned}
$$

and $U_{+}(x, y)$ and $U_{-}(x, y)$ are the restrictions of $U(x, y)$ to $\Omega_{+}^{0}$ and $\Omega_{-}^{0}$.

3. Suppose that $D_{x}^{k} D_{y}^{l} U=U^{(k, l)}$ coincides with $D_{x}^{k} D_{y}^{l} U_{ \pm}$in $\Omega_{ \pm}^{0}$ respectively. It is required that

$$
\int_{0}^{1}\left(\int_{0}^{1}\left|U^{(k, l)}\right| d x\right)^{2} d y+\int_{0}^{1}\left(\int_{0}^{1}\left|U^{(k, l)}\right| d y\right)^{2} d x<\infty
$$

for all $k$ and $l$ such that $k+l=2 m$.

When studying the Lyapunov equation for operators with local boundary conditions, Belonosov [1], [2] looked for a solution of (1.2) in the form (2.1) with a kernel which satisfies conditions $1-3$. In [3] he obtained a number of properties of the function $U(x, y)$ which can be extended to the present case. In particular, he proved that the traces of the derivatives $D_{x}^{k} D_{y}^{l}$ of order $k+l=2 m-1$ on the line $\gamma=\{x=y:(x, y) \in \Omega\}$ belong to $L_{2}\left(\gamma, \mathbb{C}_{n \times n}\right)$ and

$$
\begin{aligned}
& \left.D_{x}^{2 m-k-1} D_{y}^{k} U_{+}\right|_{y=x}-\left.D_{x}^{2 m-k-1} D_{y}^{k} U_{-}\right|_{y=x} \\
& \quad=(-1)^{k}\left[\left.D_{x}^{2 m-1} U_{+}\right|_{y=x}-\left.D_{x}^{2 m-1} U_{-}\right|_{y=x}\right], \quad 0 \leq k \leq 2 m-1 .
\end{aligned}
$$


For all $\alpha \in \omega$ and $k+l=2 m-1$ the traces of the derivatives $D_{x}^{k} D_{y}^{l} U$ on the lines $x=\alpha$ belong to $L_{2}\left(\omega, \mathbb{C}_{n \times n}\right)$ with respect to the variable $y$. The mapping $\alpha \mapsto D_{x}^{k} D_{y}^{l} U(\alpha, y)$, defined on $\omega$ and taking values in $L_{2}\left(\omega, \mathbb{C}_{n \times n}\right)$, is uniformly continuous. In an analogous way we find that $\mathbf{U}$ is a solution of (1.2) if:

I. $U_{+}(x, y)$ and $U_{-}(x, y)$ are solutions of

$$
\mathbf{A}^{*}\left(x, D_{x}\right) U(x, y)+\left[\overline{\mathbf{A}^{*}}\left(y, D_{y}\right) U^{T}(x, y)\right]^{T}=0
$$

in the domains $\Omega_{+}^{0}$ and $\Omega_{-}^{0}$.

II. $U(x, y)$ satisfies the boundary conditions

$$
\begin{aligned}
& \mathbf{B}_{i 1}\left(D_{x}\right) U(x, y)+\mathbf{B}_{i 2}\left(D_{x}\right) U(x, y)=0, \\
& \mathbf{C}_{i 1}\left(D_{y}\right) U(x, y)+\mathbf{C}_{i 2}\left(D_{y}\right) U(x, y)=0,
\end{aligned}
$$

where

$$
\begin{aligned}
& \mathbf{B}_{i 1}\left(D_{x}\right) U(x, y)=\widetilde{B}_{i}^{1} U_{x}^{\left(k_{i}\right)}(0, y)+\sum_{j=0}^{k_{i}-1} \widetilde{B}_{i j}^{1} U_{x}^{(j)}(0, y), \\
& \mathbf{B}_{i 2}\left(D_{x}\right) U(x, y)=\widetilde{B}_{i}^{2} U_{x}^{\left(k_{i}\right)}(1, y)+\sum_{j=0}^{k_{i}-1} \widetilde{B}_{i j}^{2} U_{x}^{(j)}(1, y) . \\
& \mathbf{C}_{i 1}\left(D_{y}\right) U(x, y)=U_{y}^{\left(k_{i}\right)}(x, 0) \widetilde{B}_{i}^{1 *}+\sum_{j=0}^{k_{i}-1} U_{y}^{(j)}(x, 0) \widetilde{B}_{i j}^{1 *}, \\
& \mathbf{C}_{i 2}\left(D_{y}\right) U(x, y)=U_{y}^{\left(k_{i}\right)}(x, 1) \widetilde{B}_{i}^{2 *}+\sum_{j=0}^{k_{i}-1} U_{y}^{(j)}(x, 1) \widetilde{B}_{i j}^{2 *}, \quad i=1, \ldots, 2 m .
\end{aligned}
$$

III. $\left.D_{x}^{2 m-1} U_{+}\right|_{y=x}-\left.D_{x}^{2 m-1} U_{-}\right|_{y=x}=\frac{1}{2} \mathbf{E}$, where $\mathbf{E}$ is the identity matrix in $\mathbb{C}_{n \times n}$.

Thus we have obtained the boundary value problem I-III with respect to $U(x, y)$. Henceforth we omit the tilde sign over the coefficients in boundary conditions.

This boundary value problem can be modified by getting rid of condition III. It is not difficult to construct $T(x, y)$ which belongs to $C^{2 m+\varepsilon}\left(\bar{\Omega}_{ \pm}^{0}\right) \cap$ $C^{2 m-2+\varepsilon}\left(\bar{\Omega}, \mathbb{C}_{n \times n}\right)$ and satisfies III on the diagonal $x=y$. So $H(x, y)=$ $U(x, y)-T(x, y)$ satisfies $1-3$, belongs to $W_{2}^{2 m}$ in the domain $\Omega_{\delta}=\{(x, y) \in$ $\Omega:|x-y|<1-\delta, \delta<(x+y) / 2<1-\delta, \delta>0\}$ and is a solution of the boundary value problem

$$
\begin{gathered}
\mathbf{A}_{1} H(x, y)+\left[\mathbf{A}_{2} H^{T}(x, y)\right]^{T}=F(x, y), \quad(x, y) \in \Omega, \\
\mathbf{B}_{i 1}\left(D_{x}\right) H(x, y)+\mathbf{B}_{i 2}\left(D_{x}\right) H(x, y)=\varphi_{i}(y), \quad i=1, \ldots, 2 m, \\
\mathbf{C}_{j 1}\left(D_{y}\right) H(x, y)+\mathbf{C}_{j 2}\left(D_{y}\right) H(x, y)=\psi_{j}(x), \quad j=1, \ldots, 2 m,
\end{gathered}
$$


where $\mathbf{A}_{1}=\mathbf{A}^{*}\left(x, D_{x}\right), \mathbf{A}_{2}=\overline{\mathbf{A}}^{*}\left(y, D_{y}\right)$ and $F(x, y), \varphi_{i}(y), \psi_{j}(x)$ are obviously determined by $T(x, y)$ and belong to $C^{\varepsilon}\left(\Omega, \mathbb{C}_{n \times n}\right), C^{2 m-k_{i}+\varepsilon}\left(\omega, \mathbb{C}_{n}\right)$, $C^{2 m-k_{j}+\varepsilon}\left(\omega, \mathbb{C}_{n}\right), 0<\varepsilon<1$, respectively.

3. Compatibility conditions. To solve the boundary value problem (2.3)-(2.5), we first construct $Q(x, y)$ which satisfies the boundary conditions (2.4)-(2.5). Then the difference $H-Q$ satisfies the boundary value problem with homogeneous boundary conditions. Assume that $Q \in C^{k}\left(\bar{\Omega}, \mathbb{C}_{n \times n}\right)$. Then $Q(x, y)$ satisfies the following equalities:

$$
\begin{aligned}
& {\left[\mathbf{B}_{i 1}\left(D_{x}\right)+\mathbf{B}_{i 2}\left(D_{x}\right)\right]\left[\mathbf{C}_{j 1}\left(D_{y}\right)+\mathbf{C}_{j 2}\left(D_{y}\right)\right] Q(x, y)} \\
& \quad=\left[\mathbf{C}_{j 1}\left(D_{y}\right)+\mathbf{C}_{j 2}\left(D_{y}\right)\right]\left[\mathbf{B}_{i 1}\left(D_{x}\right)+\mathbf{B}_{i 2}\left(D_{x}\right)\right] Q(x, y), \quad i, j=1, \ldots, 2 m,
\end{aligned}
$$

where we insert $Q(x, y)$ only in the equalities in which the total order of the boundary conditions on the left and right sides of (3.1) does not exceed $k$. Hence for the right sides of (2.4), (2.5) we have

$$
\left[\mathbf{B}_{i 1}\left(D_{x}\right)+\mathbf{B}_{i 2}\left(D_{x}\right)\right] \psi_{j}(x)=\left[\mathbf{C}_{j 1}\left(D_{y}\right)+\mathbf{C}_{j 2}\left(D_{y}\right)\right] \varphi_{i}(y) .
$$

Henceforth we call (3.1) the compatibility conditions of order $k$. Notice that

$$
\begin{aligned}
\varphi_{i}(y) & =-\left[\mathbf{B}_{i 1}\left(D_{x}\right)+\mathbf{B}_{i 2}\left(D_{x}\right)\right] T(x, y), \\
\psi_{j}(x) & =-\left[\mathbf{C}_{j 1}\left(D_{y}\right)+\mathbf{C}_{j 2}\left(D_{y}\right)\right] T(x, y) .
\end{aligned}
$$

We have $T \in C^{2 m-2+\varepsilon}\left(\bar{\Omega}, \mathbb{C}_{n \times n}\right)$ and the derivatives of order $2 m-1$ have a discontinuity on the diagonal. Therefore $\varphi_{i}(y)$ and $\psi_{j}(x)$ satisfy the compatibility conditions of order at most $2 m-2$. Let us modify the boundary value problem (2.3)-(2.5) in such a way that $\varphi_{i}(y)$ and $\psi_{j}(x)$ satisfy the compatibility conditions of order $2 m-1$.

We construct a solution of the following boundary value problem with local boundary conditions in the quadrant:

$$
\begin{aligned}
& v_{i x}^{(2 m)}(x, y)+v_{i y}^{(2 m)}(x, y)=0, \quad x>0, y>0, \\
& v_{1 y}^{\left(k_{j}\right)}(x, 0) B_{j}^{1 *}+(-1)^{k_{j}} v_{4 y}^{\left(k_{j}\right)}(x, 0) B_{j}^{2 *}=\frac{\theta_{j}}{\left(2 m-k_{j}-1\right) !} x^{2 m-k_{j}-1}, \\
& v_{2 y}^{\left(k_{j}\right)}(x, 0) B_{j}^{1 *}+(-1)^{k_{j}} v_{3 y}^{\left(k_{j}\right)}(x, 0) B_{j}^{2 *}=\frac{\theta_{j+2 m}}{\left(2 m-k_{j}-1\right) !} x^{2 m-k_{j}-1}, \\
& B_{i}^{1} v_{1 x}^{\left(k_{i}\right)}(0, y)+(-1)^{k_{i}} B_{i}^{2} v_{2 x}^{\left(k_{i}\right)}(0, y)=\frac{\sigma_{i}}{\left(2 m-k_{i}-1\right) !} y^{2 m-k_{i}-1}, \\
& B_{i}^{1} v_{4 x}^{\left(k_{i}\right)}(0, y)+(-1)^{k_{i}} B_{i}^{2} v_{3 x}^{\left(k_{i}\right)}(0, y)=\frac{\sigma_{i+2 m}}{\left(2 m-k_{i}-1\right) !} y^{2 m-k_{i}-1}, \\
& i, j=1, \ldots, 2 m,
\end{aligned}
$$

where $\theta_{l}, \sigma_{l}, l=1, \ldots, 4 m$, are arbitrary elements of $\mathbb{C}_{n \times n}$, and $v_{s}(x, y)$, $s=1, \ldots, 4$, take values in $\mathbb{C}_{n \times n}$. 
Passing to polar coordinates and using the method of separation of variables one can show [9] that (3.2) has a particular solution of the form

$$
v_{s}(r, \varphi)=r^{2 m-1} \sum_{k=0}^{\nu}(\ln r)^{k} \chi_{s k}(\varphi),
$$

where $\chi_{s k} \in C^{\infty}[0,2 \pi]$.

Write $v_{s}$ in Cartesian coordinates and apply the following transformations:

$$
\begin{array}{ll}
\check{v}_{1}(x, y)=v_{1}(x, y), & \check{v}_{2}(x, y)=v_{2}(1-x, y), \\
\check{v}_{3}(x, y)=v_{3}(1-x, 1-y), & \check{v}_{4}(x, y)=v_{4}(x, 1-y) .
\end{array}
$$

Further, multiply $\check{v}_{s}(x, y), s=1, \ldots, 4$, by cut-off functions $h_{s}$ which have the following properties:

1. $h_{1} \equiv 1$ in the $\varepsilon_{1}$-neighborhood of $(0,0), h_{2} \equiv 1$ in the $\varepsilon_{1}$-neighborhood of $(1,0), h_{3} \equiv 1$ in the $\varepsilon_{1}$-neighborhood of $(1,1), h_{4} \equiv 1$ in the $\varepsilon_{1}$-neighborhood of $(0,1)$,

2. $h_{s} \equiv 0$ outside the $\varepsilon_{2}$-neighborhood of the corresponding angular points, where $\varepsilon_{2}>\varepsilon_{1}$.

Notice that after these transformations each of the functions $h_{s} \check{v}_{s}$ satisfies $\left(h_{s} \check{v}_{s}\right)_{x}^{(2 m)}+\left(h_{s} \check{v}_{s}\right)_{y}^{(2 m)}=0$ in the $\varepsilon_{1}$-neighborhood of the corresponding angular point.

Set

$$
v(x, y)=\sum_{s=1}^{4} h_{s}(x, y) \check{v}_{s}(x, y) .
$$

THEOREM 1. Let

$$
\begin{aligned}
\check{\varphi}_{i}(y) & =\varphi_{i}(y)-\left(\mathbf{B}_{i 1}\left(D_{x}\right)+\mathbf{B}_{i 2}\left(D_{x}\right)\right) v(x, y), & & i=1, \ldots, 2 m, \\
\check{\psi}_{j}(x) & =\psi_{j}(x)-\left(\mathbf{C}_{j 1}\left(D_{y}\right)+\mathbf{C}_{j 2}\left(D_{y}\right)\right) v(x, y), & & j=1, \ldots, 2 m .
\end{aligned}
$$

Then one can select $\theta_{l}$ and $\sigma_{l}, l=1, \ldots, 4 m$, (from (3.2)) so that the functions $\check{\varphi}_{i}(x, y)$ and $\check{\psi}_{j}(x, y)$ satisfy the compatibility conditions (3.1).

Consider the boundary value problem $(2.3)-(2.5)$ for $H(x, y)-v(x, y)$. Then the compatibility conditions (3.1) will appear in the following way:

$$
\begin{aligned}
{\left[\mathbf{C}_{j 1}\left(D_{y}\right)+\right.} & \left.\mathbf{C}_{j 2}\left(D_{y}\right)\right] \varphi_{i}(y) \\
& -\left[\mathbf{C}_{j 1}\left(D_{y}\right)+\mathbf{C}_{j 2}\left(D_{y}\right)\right]\left[\mathbf{B}_{i 1}\left(D_{x}\right)+\mathbf{B}_{i 2}\left(D_{x}\right)\right] v(x, y) \\
= & {\left[\mathbf{B}_{i 1}\left(D_{x}\right)+\mathbf{B}_{i 2}\left(D_{x}\right)\right] \psi_{j}(x) } \\
& -\left[\mathbf{B}_{i 1}\left(D_{x}\right)+\mathbf{B}_{i 2}\left(D_{x}\right)\right]\left[\mathbf{C}_{j 1}\left(D_{y}\right)+\mathbf{C}_{j 2}\left(D_{y}\right)\right] v(x, y) .
\end{aligned}
$$

The function $v(x, y)$ belongs to $W_{2}^{2 m-1+\varepsilon}\left(\Omega, \mathbb{C}_{n \times n}\right)$ and vanishes together with the derivatives $D_{x}^{l} D_{y}^{k} v, k+l \leq 2 m-2$, at the angular points. Taking into account that $\varphi_{i}(y)$ and $\psi_{j}(x)$ satisfy the compatibility conditions of 
order $2 m-2$, we conclude that (3.4) can be represented in the following way:

$$
\begin{aligned}
\sigma_{i} B_{j}^{1 *}+\sigma_{i+2 m} & B_{j}^{2 *}-B_{i}^{1} \theta_{j}-B_{i}^{2} \theta_{j+2 m} \\
= & {\left[\mathbf{B}_{i 1}\left(D_{x}\right)+\mathbf{B}_{i 2}\left(D_{x}\right)\right] \psi_{j}(x) } \\
& -\left[\mathbf{C}_{j 1}\left(D_{y}\right)+\mathbf{C}_{j 2}\left(D_{y}\right)\right] \varphi_{i}(y), \quad k_{i}+k_{j}=2 m-1,
\end{aligned}
$$

where $\theta_{l}, \sigma_{l}, l=1, \ldots, 4 m$, are functions with values in $\mathbb{C}_{n \times n}$. From the regularity of the boundary conditions $(2.4),(2.5)$ it follows that the matrix $\left(B_{i}^{1} B_{i}^{2}\right)$ has maximal rank and therefore (3.5) is solvable with respect to $\theta_{l}$, $\sigma_{l}, l=1, \ldots, 4 m$. Calculating $\theta_{l}, \sigma_{l}$, we satisfy the compatibility conditions (3.1). The theorem is proved.

Consider the difference $H(x, y)-v(x, y)=H_{1}(x, y)$. Replacing $H$ in (2.3)-(2.5) by $H_{1}(x, y)+v(x, y)$ we obtain

$$
\begin{aligned}
\mathbf{A}_{1} H_{1}(x, y)+\left[\mathbf{A}_{2} H_{1}^{T}(x, y)\right]^{T}=\widetilde{F}(x, y), & \\
\mathbf{B}_{i 1}\left(D_{x}\right) H_{1}+\mathbf{B}_{i 2}\left(D_{x}\right) H_{1}=\check{\varphi}_{i}(y), & i=1, \ldots, 2 m, \\
\mathbf{C}_{j 1}\left(D_{y}\right) H_{1}+\mathbf{C}_{j 2}\left(D_{y}\right) H_{1}=\check{\psi}_{j}(x), & j=1, \ldots, 2 m .
\end{aligned}
$$

The right sides in (3.7), (3.8) satisfy the compatibility conditions (3.1) distinct from those satisfied by the right sides in (2.4), (2.5). By the special choice of $v(x, y)$, in the neighborhood of all angular points we have $v_{x}^{(2 m)}+$ $v_{y}^{(2 m)}(x, y)=0$, therefore $\widetilde{F} \in W_{2}^{\varepsilon}(\Omega), \check{\varphi}_{i} \in W_{2}^{2 m-k_{i}-1 / 2+\varepsilon}\left(\omega, \mathbb{C}_{n \times n}\right)$, $\check{\psi}_{j} \in W_{2}^{2 m-k_{j}-1 / 2+\varepsilon}\left(\omega, \mathbb{C}_{n \times n}\right), \varepsilon<1 / 2$.

4. Transformation of the nonhomogeneous boundary value problem to the homogeneous one. We proceed to construct $V \in$ $W_{2}^{2 m+\varepsilon}\left(\Omega, \mathbb{C}_{n \times n}\right)$ which satisfies (3.7), (3.8). First we construct a function $\widetilde{V}(x, y)=V_{1}(x, y)+V_{2}(x, y)$ in the case when the right sides in (3.7), (3.8) satisfy

$$
\begin{array}{ll}
\check{\varphi}_{i}^{(p)}(0)=\check{\varphi}_{i}^{(p)}(1)=0, & p=0, \ldots, 2 m-k_{i}-1, \quad i=1, \ldots, 2 m, \\
\check{\psi}_{j}^{(p)}(0)=\check{\psi}_{j}^{(p)}(1)=0, & p=0, \ldots, 2 m-k_{j}-1, \quad j=1, \ldots, 2 m,
\end{array}
$$

where $V_{1}(x, y)$ satisfies the boundary conditions of the type

$$
\begin{aligned}
& B_{i}^{1} V_{1 x}^{\left(k_{i}\right)}(0, y)+\sum_{p=0}^{k_{i}-1} B_{i p}^{1} V_{1 x}^{(p)}(0, y) \\
& \quad+B_{i}^{2} V_{1 x}^{\left(k_{i}\right)}(1, y)+\sum_{p=0}^{k_{i}-1} B_{i p}^{2} V_{1 x}^{(p)}(1, y)=\check{\varphi}_{i}(y), \quad i=1, \ldots, 2 m,
\end{aligned}
$$


[cont.]

$$
\begin{aligned}
& V_{1 y}^{\left(k_{j}\right)}(x, 0) B_{j}^{1 *}+\sum_{p=0}^{k_{j}-1} V_{1 y}^{(p)}(x, 0) B_{j p}^{1 *} \\
& \quad+V_{1 y}^{\left(k_{j}\right)}(x, 1) B_{j}^{2 *}+\sum_{p=0}^{k_{j}-1} V_{1 y}^{(p)}(x, 1) B_{j p}^{2 *}=0, \quad j=1, \ldots, 2 m,
\end{aligned}
$$

and $V_{2}(x, y)$ satisfies the boundary conditions of the type

$$
\begin{aligned}
B_{i}^{1} V_{2 x}^{\left(k_{i}\right)}(0, y) & +\sum_{p=0}^{k_{i}-1} B_{i p}^{1} V_{2 x}^{(p)}(0, y) \\
& +B_{i}^{2} V_{2 x}^{\left(k_{i}\right)}(1, y)+\sum_{p=0}^{k_{i}-1} B_{i p}^{2} V_{2 x}^{(p)}(1, y)=0, \quad i=1, \ldots, 2 m
\end{aligned}
$$

$$
\begin{aligned}
& V_{2 y}^{\left(k_{j}\right)}(x, 0) B_{j}^{1 *}+\sum_{p=0}^{k_{j}-1} V_{2 y}^{(p)}(x, 0) B_{j p}^{1 *} \\
& \quad+V_{2 y}^{\left(k_{j}\right)}(x, 1) B_{j}^{2 *}+\sum_{p=0}^{k_{j}-1} V_{2 y}^{(p)}(x, 1) B_{j p}^{2 *}=\check{\psi}_{j}(x), \quad j=1, \ldots, 2 m .
\end{aligned}
$$

Let $\check{\varphi}_{i}(y) \equiv 0$ when $y \in(-\infty, 0) \cup(1, \infty), \check{\psi}_{j}(x) \equiv 0$ when $x \in(-\infty, 0) \cup$ $(1, \infty), i, j=1, \ldots, 2 m$. By the regularity of the boundary conditions, (4.2) is solvable with respect to $V_{1 y}^{\left(k_{j}\right)}(x, 0), V_{1 y}^{\left(k_{j}\right)}(x, 1), V_{1 x}^{\left(k_{i}\right)}(0, y), V_{1 x}^{\left(k_{i}\right)}(1, y)$, $i, j=1, \ldots, 2 m$. So one can deduce that $V_{1}(x, y)$ takes the following values on the boundary of $\Omega$ :

$$
\begin{aligned}
& V_{1 y}^{(l)}(x, 0)=V_{1 y}^{(l)}(x, 1)=0, \quad V_{1 x}^{(l)}(0, y)=\varphi^{l}(y), \\
& V_{1 x}^{(l)}(1, y)=\varphi^{l+2 m}(y), \quad l=0, \ldots, 2 m-1,
\end{aligned}
$$

where $\varphi^{l}(y), \varphi^{l+2 m}(y), l=0, \ldots, 2 m-1$, are linear combinations of $\check{\varphi}_{i}(y)$, $i=1, \ldots, 2 m$. If the boundary condition of order $k_{s}$ is absent then, for simplicity, we assume $\varphi^{s}=\varphi^{s+2 m}=0$.

We consider a model problem. We have to find $u_{1} \in W_{2}^{2 m+\varepsilon}\left(\Omega_{1}^{N}\right)$, where $\Omega_{1}^{N}=\{(x, y): 0<x<N, 0<y<N\}$, which takes given values on the boundary of $\Omega_{1}=\{(x, y): x>0, y>0\}$ :

$$
u_{1 y}^{(l)}=0, \quad u_{1 x}^{(l)}=\varphi^{l}(y), \quad l=0, \ldots, 2 m-1 .
$$

Let

$$
u_{(\varphi)}(x, y)=\frac{1}{x} \int_{0}^{\infty} \omega\left(\frac{y-\xi}{x}\right) \varphi(\xi) d \xi,
$$

where $\omega \in C_{0}^{\infty}\left(\mathbb{R}_{+}\right)$and $\int_{0}^{\infty} \omega d x=1$. Let us prove the following 
LEMmA 1. If $\varphi \in W_{2}^{2 m-1 / 2-k+\varepsilon}\left(\mathbb{R}_{+}\right)$then $x^{k} u_{(\varphi)}(x, y) \in W_{2}^{2 m+\varepsilon}\left(\Omega_{1}^{N}\right)$, $k=0, \ldots, 2 m-1$, for any $N>0$ where $\varphi^{(l)}(0)=0, l=0, \ldots, 2 m-1-k$.

Proof. First we show that

$$
\left\|\frac{\partial^{2 m}\left(x^{k} u_{(\varphi)}(x, y)\right)}{\partial y^{2 m}}\right\|_{L_{2}\left(\Omega_{1}\right)} \leq C\|\varphi\|_{W_{2}^{2 m-1 / 2-k}\left(\mathbb{R}_{+}\right)},
$$

where $\varphi \in C_{0}^{\infty}\left(\mathbb{R}_{+}\right)$. In fact

$$
\begin{aligned}
\left\|\frac{\partial^{2 m}\left(x^{k} u_{(\varphi)}(x, y)\right)}{\partial y^{2 m}}\right\|_{L_{2}\left(\Omega_{1}\right)} \\
=\left\|\frac{\partial^{2 m}}{\partial y^{2 m}}\left(x^{k} \int_{0}^{\infty} \omega(t) \varphi(y-x t) d t\right)\right\|_{L_{2}\left(\Omega_{1}\right)} \\
=\left\|\frac{\partial^{k+1}}{\partial y^{k+1}}\left(x^{k-1} \int_{0}^{\infty} \omega\left(\frac{y-\xi}{x}\right) \varphi^{(2 m-1-k)}(\xi) d \xi\right)\right\|_{L_{2}\left(\Omega_{1}\right)} \\
=\left\|\frac{1}{x} \int_{0}^{\infty} \omega^{(k+1)}(t) \varphi^{(2 m-1-k)}(y-x t) d t\right\|_{L_{2}\left(\Omega_{1}\right)}
\end{aligned}
$$

Let us add and subtract $\varphi^{(2 m-1-k)}(y)$ in the last integral. Then one can represent (4.5) in the following way:

$$
\begin{aligned}
& \| \frac{1}{x} \int_{0}^{\infty} \omega^{(k+1)}(t)\left[\varphi^{(2 m-1-k)}(y-x t)-\varphi^{(2 m-1-k)}(y)\right] d t \\
& \quad+\frac{1}{x} \int_{0}^{\infty} \omega^{(k+1)}(t) \varphi^{(2 m-1-k)}(y) d t \|_{L_{2}\left(\Omega_{1}\right)} \\
& =\left\|\frac{1}{x} \int_{0}^{\infty} \omega^{(k+1)}(t)\left[\varphi^{(2 m-1-k)}(y-x t)-\varphi^{(2 m-1-k)}(y)\right] d t\right\|_{L_{2}\left(\Omega_{1}\right)},
\end{aligned}
$$

since

$$
\frac{1}{x} \int_{0}^{\infty} \omega^{(k+1)}(t) \varphi^{(2 m-1-k)}(y) d t=\frac{\varphi^{(2 m-1-k)}(y)}{x} \int_{0}^{\infty} d\left(\omega^{(k)}(t)\right) d t=0,
$$

due to the fact that $\omega \in C_{0}^{\infty}\left(\mathbb{R}_{+}\right)$. Without loss of generality we assume that $\omega \in C_{0}^{\infty}[0,1]$. Let $\tau=x t$. Then

$$
\left\|\frac{1}{x} \int_{0}^{\infty} \omega^{(k+1)}(t)\left[\varphi^{(2 m-1-k)}(y-x t)-\varphi^{(2 m-1-k)}(y)\right] d t\right\|_{L_{2}\left(\Omega_{1}\right)}
$$




$$
\begin{gathered}
=\left(\int_{0}^{\infty} \int_{0}^{\infty}\left|\frac{1}{x} \int_{0}^{1} \omega^{(k+1)}(t)\left[\varphi^{(2 m-1-k)}(y-x t)-\varphi^{(2 m-1-k)}(y)\right] d t\right|^{2} d x d y\right)^{1 / 2} \\
\leq C\left(\int_{0}^{\infty} d y \int_{0}^{\infty} d x\left|\frac{1}{x^{2}} \int_{0}^{x} \tau^{\alpha} \frac{\varphi^{(2 m-1-k)}(y-\tau)-\varphi^{(2 m-1-k)}(y)}{\tau^{\alpha}} d \tau\right|^{2}\right)^{1 / 2} \\
\leq C\left(\int _ { 0 } ^ { \infty } d y \int _ { 0 } ^ { \infty } d x \left(\frac{1}{x^{4}} \int_{0}^{x} \tau^{2 \alpha} d \tau\right.\right. \\
\left.\left.\quad \times \int_{0}^{x} \frac{\left|\varphi^{(2 m-1-k)}(y-\tau)-\varphi^{(2 m-1-k)}(y)\right|^{2}}{|\tau|^{2 \alpha}} d \tau\right)\right)^{1 / 2},
\end{gathered}
$$

where $\alpha<1$. Since

$$
\frac{1}{x^{4}} \int_{0}^{x} \tau^{2 \alpha} d \tau=\frac{1}{2 \alpha+1} x^{2 \alpha-3}
$$

after changing the limits of integration in the last two integrals in (4.6) one can obtain

$$
\begin{aligned}
& C\left(\int _ { 0 } ^ { \infty } d y \int _ { 0 } ^ { \infty } d x \left(\frac{1}{x^{4}} \int_{0}^{x} \tau^{2 \alpha} d \tau\right.\right. \\
& \left.\left.\quad \times \int_{0}^{x} \frac{\left|\varphi^{(2 m-1-k)}(y-\tau)-\varphi^{(2 m-1-k)}(y)\right|^{2}}{|\tau|^{2 \alpha}} d \tau\right)\right)^{1 / 2} \\
\leq & C_{1}\left(\int_{0}^{\infty} d y \int_{0}^{\infty} x^{2 \alpha-3} d x \int_{0}^{x} \frac{\left|\varphi^{(2 m-1-k)}(y-\tau)-\varphi^{(2 m-1-k)}(y)\right|^{2}}{|\tau|^{2 \alpha}} d \tau\right)^{1 / 2} \\
\leq & C_{1}\left(\int_{0}^{\infty} d y \int_{0}^{\infty} \frac{\left|\varphi^{(2 m-1-k)}(y-\tau)-\varphi^{(2 m-1-k)}(y)\right|^{2}}{|\tau|^{2 \alpha}} d \tau \int_{\tau}^{\infty} x^{2 \alpha-3} d x\right)^{1 / 2} \\
\leq & C_{2}\left(\int_{0}^{\infty} \int_{0}^{\infty} \frac{\left|\varphi^{(2 m-1-k)}(y-\tau)-\varphi^{(2 m-1-k)}(y)\right|^{2}}{|\tau|^{2}} d y d \tau\right)^{1 / 2} \\
\leq & C_{2}\left\|\varphi^{(2 m-1 / 2-k)}\right\|_{W_{2}^{1 / 2}\left(\mathbb{R}_{+}\right)^{.}}
\end{aligned}
$$

From (4.7) it follows that

$$
\left\|\frac{\partial^{2 m}\left(x^{k} u_{(\varphi)}(x, y)\right)}{\partial y^{2 m}}\right\|_{L_{2}\left(\Omega_{1}\right)} \leq C\|\varphi\|_{W_{2}^{2 m-1 / 2-k}\left(\mathbb{R}_{+}\right)} .
$$

Consider the derivative of order $2 m$ with respect to $x$. From the formulas 
for differentiation of the product and of the composite function we have

$$
\begin{aligned}
& \frac{\partial^{2 m}\left(x^{k} u_{(\varphi)}(x, y)\right)}{\partial x^{2 m}}=\sum_{p=0}^{k} \sum_{q=0}^{k-p} \sum_{r=0}^{k-p-q}\left\{a_{p} b_{q} c_{r} \frac{1}{x}\right. \\
& \times {\left[\int_{0}^{\infty} \omega^{(k-p-q-r+1)}(t) t^{2 m-p-q-r} \varphi^{(2 m-1-k)}(y-x t) d t\right.} \\
&\left.\left.-(2 m-p-q-r) \int_{0}^{\infty} \omega^{(k-p-q-r)}(t) t^{2 m-p-q-r-1} \varphi^{(2 m-1-k)}(y-x t) d t\right]\right\} \\
&-\frac{1}{x}\left[(2 m-k) \int_{0}^{\infty} \omega(t) t^{2 m-1-k} \varphi^{(2 m-1-k)}(y-x t) d t\right. \\
&\left.+\int_{0}^{\infty} \omega^{\prime}(t) t^{2 m-k} \varphi^{(2 m-1-k)}(y-x t) d t\right],
\end{aligned}
$$

where the constants $a_{p}, b_{q}, c_{r}$ can be easily calculated. Taking into account that the singularity with respect to $x$ in (4.9) is the same as in (4.5) and that

$$
\begin{aligned}
(2 m-p-q-r) \int_{0}^{\infty} \omega^{(k-p-q-r)}(t) & t^{2 m-1-p-q-r} d t \\
& +\int_{0}^{\infty} \omega^{(k-p-q-r-1)}(t) t^{2 m-p-q-r} d t=0
\end{aligned}
$$

for all admissible $k, p, q, r$, we obtain

$$
\left\|\frac{\partial^{2 m}\left(x^{k} u_{(\varphi)}(x, y)\right)}{\partial x^{2 m}}\right\|_{L_{2}\left(\Omega_{1}\right)} \leq C\|\varphi\|_{W_{2}^{2 m-1 / 2-k}\left(\mathbb{R}_{+}\right)} .
$$

It is clear that all the derivatives of order less than $2 m$ of the function $x^{k} u_{\varphi}$ belong to $L_{2}\left(\Omega_{1}^{N}\right)$. Here we write $\Omega_{1}^{N}$ instead of $\Omega_{1}$ because the functions $D_{x}^{p} D_{y}^{q}\left(x^{k} u_{\varphi}\right), p+q<2 m$, have singularities at infinity. From (4.8), (4.10) it follows [14] that

$$
\left\|x^{k} u_{(\varphi)}(x, y)\right\|_{W_{2}^{2 m}\left(\Omega_{1}^{N}\right)} \leq C_{N}\|\varphi\|_{W_{2}^{2 m-1 / 2-k}\left(\mathbb{R}_{+}\right)} .
$$

Now if $\varphi \in W_{2}^{2 m-1 / 2-k}\left(\mathbb{R}_{+}\right)$, then we assume that

$$
\varphi^{(p)}(0)=0, \quad p=0, \ldots, l-2, \quad \int_{0}^{1}\left|\varphi^{(l-1)}(\xi)\right| \frac{d \xi}{\xi}<\infty,
$$

where $l=2 m-k$. From (4.11) it follows that $\varphi(\xi)$ can be approximated by functions $\zeta_{l} \in C_{0}^{\infty}\left(\mathbb{R}_{+}\right)$,

$$
\left\|\varphi-\zeta_{l}\right\|_{W_{2}^{2 m-1 / 2-k}\left(\mathbb{R}_{+}\right)} \rightarrow 0, \quad l \rightarrow \infty .
$$


From (4.12) and the considerations above it follows that

$$
\left\|x^{k} u_{(\varphi)}(x, y)\right\|_{W_{2}^{2 m}\left(\Omega_{1}^{N}\right)} \leq C_{N}\|\varphi\|_{W_{2}^{2 m-1 / 2-k}\left(\mathbb{R}_{+}\right)} .
$$

Analogously one can obtain the estimate

$$
\left\|x^{k} u_{(\varphi)}(x, y)\right\|_{W_{2}^{2 m+1}\left(\Omega_{1}^{N}\right)} \leq C_{N}\|\varphi\|_{W_{2}^{2 m+1 / 2-k}\left(\mathbb{R}_{+}\right)},
$$

where $\varphi \in W_{2}^{2 m+1 / 2-k}\left(\mathbb{R}_{+}\right)$and the necessary compatibility conditions when $\xi=0$ are satisfied. Using the results of Grisvard concerning interpolation spaces ([8], Theorems 7.3 and 7.6) we obtain

$$
\left\|x^{k} u_{(\varphi)}(x, y)\right\|_{W_{2}^{2 m+\varepsilon}\left(\Omega_{1}^{N}\right)} \leq C_{N}\|\varphi\|_{W_{2}^{2 m-1 / 2-k+\varepsilon}\left(\mathbb{R}_{+}\right)},
$$

where $\varphi^{(l)}(0)=0, l=0, \ldots, 2 m-1-k$. The lemma is proved.

Let

$$
u_{1}(x, y)=u_{\left(\varphi^{0}\right)}(x, y)+\sum_{k=1}^{2 m-1} x^{k} u_{\left(\check{\varphi}^{k}\right)}(x, y),
$$

where

$$
\check{\varphi}^{k}=\frac{1}{k !}\left[\varphi^{k}-\frac{\partial^{k} u_{\left(\varphi^{0}\right)}}{\partial x^{k}}(0, y)-\sum_{l=1}^{k-1} \frac{\partial^{k}\left(x^{l} u_{\left(\check{\varphi}^{l}\right)}\right)}{\partial x^{k}}(0, y)\right] .
$$

From the previous section and the definition of $\varphi^{0}, \check{\varphi}^{k}, k=1, \ldots, 2 m-1$, it follows that $\varphi^{0} \in W_{2}^{2 m-1 / 2+\varepsilon}\left(\mathbb{R}_{+}\right), \check{\varphi}^{k} \in W_{2}^{2 m-1 / 2-k+\varepsilon}\left(\mathbb{R}_{+}\right)$. From Lemma 1 we find that $u_{1} \in W_{2}^{2 m+\varepsilon}\left(\Omega_{1}^{N}\right)$. Furthermore $u_{1 y}^{(k)}(x, 0)=0, k=0, \ldots$ $\ldots, 2 m-1$, since $\lim _{y \rightarrow 0} \omega^{(k)}((y-\xi) / x)=0, k=0, \ldots, 2 m-1$. It is not difficult to verify that

$$
\left.\frac{\partial^{l}}{\partial x^{l}}\left(x^{k} u_{\left(\breve{\varphi}^{k}\right)}(x, y)\right)\right|_{x=0}=0, \quad l=1, \ldots, k-1 .
$$

Therefore

$$
u_{1 x}^{(l)}(0, y)=\frac{\partial^{l} u_{\left(\varphi^{0}\right)}}{\partial x^{l}}(0, y)+\sum_{p=1}^{l} \frac{\partial^{l}\left(x^{k} u_{\left(\check{\varphi}^{k}\right)}\right)}{\partial x^{l}}(0, y) .
$$

From the formulas for differentiation of the product one can obtain

$$
\frac{\partial^{l}}{\partial x^{l}}\left(x^{l} u_{\left(\check{\varphi}^{l}\right)}(0, y)\right)=\check{\varphi}^{l}(y) .
$$

Consequently, from (4.14) and (4.15) it follows that

$$
u_{1 x}^{(l)}(0, y)=\varphi^{l}(y) .
$$

So we have shown that $u_{1}$ represented by (4.13) belongs to $W_{2}^{2 m+\varepsilon}\left(\Omega_{1}^{N}\right)$ for any $N>0$ and takes the given values (4.4). 
Define

$$
\begin{aligned}
\Omega_{2} & =\{(x, y): x<1, y>0\}, \\
\Omega_{3} & =\{(x, y): x<1, y<1\}, \\
\Omega_{4} & =\{(x, y): x>0, y<1\}, \\
\Omega_{2}^{N} & =\{(x, y):-N<x<1,0<y<N\}, \\
\Omega_{3}^{N} & =\{(x, y):-N<x<1,-N<y<1,\}, \\
\Omega_{4}^{N} & =\{(x, y): 0<x<N,-N<y<1\}
\end{aligned}
$$

and let $u_{s}, s=2,3,4$, be functions which belong to $W_{2}^{2 m+\varepsilon}\left(\Omega_{s}^{N}\right)$ and take the following values on the boundary of $\Omega_{s}, s=2,3,4$, respectively:

$$
\begin{aligned}
u_{2 y}^{(l)}(x, 0) & =0, & u_{2 x}^{(l)}(1, y) & =\varphi^{l+2 m}(y), \\
u_{3 y}^{(l)}(x, 1) & =0, & u_{3 x}^{(l)}(1, y) & =\varphi^{l+2 m}(y), \\
u_{4 y}^{(l)}(x, 1) & =0, & u_{4 y}^{(l)}(0, y) & =\varphi^{l}(y), \quad l=0, \ldots, 2 m-1 .
\end{aligned}
$$

It is not difficult to obtain $u_{s}(x, y)$ from (4.13) by a suitable change of variables.

Consider an open covering of the boundary of $\Omega$ by discs with centers at the vertices of the square and with radii $1 / 2 \leq \varepsilon \leq \sqrt{2} / 2$ and take a partition of unity subordinate to this covering,

$$
1=\sum_{s=1}^{4} \xi_{s}(x, y) \text {. }
$$

Note that it is sufficient to require the fulfillment of (4.16) only on the boundary of $\Omega$. It is clear that the function

$$
V_{1}(x, y)=\sum_{s=1}^{4} \xi_{s}(x, y) u_{s}(x, y)
$$

is the required extension. Similarly one can construct $V_{2}(x, y)$.

Due to the special selection of $V_{1}$ and $V_{2}$ the function $\widetilde{V}$ belongs to $W_{2}^{2 m+\varepsilon}\left(\Omega, \mathbb{C}_{n \times n}\right)$ and satisfies the boundary conditions $(3.7),(3.8)$, where the right sides satisfy (4.1).

We return to the original boundary problem (3.6)-(3.8) and formulate the following

THEOREM 2. If the boundary conditions (3.7), (3.8) are such that for any $k_{i}, k_{j}$ we have $k_{i}+k_{j} \geq 2 m$, then $V(x, y)$ always exists. Otherwise for the existence of $V(x, y)$ it is necessary and sufficient that the compatibility conditions (3.1) hold.

Proof. The necessity was shown in $§ 2$. To prove the sufficiency, consider (3.7), (3.8): 


$$
\begin{aligned}
B_{i}^{1} V_{x}^{\left(k_{i}\right)}(0, y) & +\sum_{p=0}^{k_{i}-1} B_{i p}^{1} V_{x}^{(p)}(0, y) \\
& +B_{i}^{2} V_{x}^{\left(k_{i}\right)}(1, y)+\sum_{p=0}^{k_{i}-1} B_{i p}^{2} V_{x}^{(p)}(1, y)=\check{\varphi}_{i}(y), \quad i=1, \ldots, 2 m, \\
V_{y}^{\left(k_{j}\right)}(x, 0) B_{j}^{1 *} & +\sum_{p=0}^{k_{j}-1} V_{y}^{(p)}(x, 0) B_{j p}^{1 *} \\
+ & V_{y}^{\left(k_{j}\right)}(x, 1) B_{j}^{2 *}+\sum_{p=0}^{k_{j}-1} V_{y}^{(p)}(x, 1) B_{j p}^{2 *}=\check{\psi}_{j}(x), \quad j=1, \ldots, 2 m .
\end{aligned}
$$

Introduce the following notations:

$$
\begin{array}{ll}
V_{x}^{(k)}(0, y)=f_{k}^{0}(y), \quad V_{y}^{(k)}(x, 0)=g_{k}^{0}(x), \\
V_{x}^{(k)}(1, y)=f_{k}^{1}(y), \quad V_{y}^{(k)}(x, 1)=g_{k}^{1}(x), \quad k=1, \ldots, 2 m-1 .
\end{array}
$$

Since we require that $V \in W_{2}^{2 m+\varepsilon}\left(\Omega, \mathbb{C}_{n \times n}\right)$, the embedding theorems yield $V \in C^{2 m-1}\left(\bar{\Omega}, \mathbb{C}_{n \times n}\right)$. Therefore the values of $f_{k}^{0}(y), f_{k}^{1}(y), g_{k}^{0}(x), g_{k}^{1}(x)$ at the vertices of the square must satisfy certain conditions, which can be written in the following way:

$$
\begin{array}{ll}
g_{l}^{0(k)}(0)=f_{k}^{0(l)}(0), & g_{l}^{0(k)}(1)=f_{k}^{1(l)}(0), \\
g_{l}^{1(k)}(0)=f_{k}^{0(l)}(1), & g_{l}^{1(k)}(1)=f_{k}^{1(l)}(1), \quad k+l \leq 2 m-1 .
\end{array}
$$

Let us successively differentiate every boundary condition in (3.7), (3.8) and add the results to the originals until the order of the boundary condition reaches $2 m-1$. Then we successively put $x=0, x=1, y=0, y=1$ in the resulting extended system of boundary conditions. In this way, we obtain the following system of equalities:

$$
\begin{aligned}
B_{i}^{1} f_{k_{i}}^{0(l)}(0)+\sum_{p=0}^{k_{i}-1} B_{i p}^{1} f_{p}^{0(l)}(0) & \\
& \quad+B_{i}^{2} f_{k_{i}}^{1(l)}(0)+\sum_{p=0}^{k_{i}-1} B_{i p}^{2} f_{p}^{1(l)}(0)=\check{\varphi}_{i}^{(l)}(0),
\end{aligned}
$$

$$
\begin{aligned}
g_{k_{j}}^{0(k)}(0) B_{j}^{1 *}+\sum_{p=0}^{k_{j}-1} g_{p}^{0(k)}(0) B_{j p}^{1 *} & \\
& \quad+g_{k_{j}}^{1(k)}(0) B_{j}^{2 *}+\sum_{p=0}^{k_{j}-1} g_{p}^{1(k)}(0) B_{j p}^{2 *}=\check{\psi}_{j}^{(k)}(0),
\end{aligned}
$$




$$
\begin{aligned}
B_{i}^{1} f_{k_{i}}^{0(l)}(1)+\sum_{p=0}^{k_{i}-1} B_{i p}^{1} f_{p}^{0(l)}(1) & \\
& \quad+B_{i}^{2} f_{k_{i}}^{1(l)}(1)+\sum_{p=0}^{k_{i}-1} B_{i p}^{2} f_{p}^{1(k)}(1)=\check{\varphi}_{i}^{(l)}(1),
\end{aligned}
$$

$$
\begin{aligned}
& g_{k_{j}}^{0(k)}(1) B_{j}^{1 *}+\sum_{p=0}^{k_{j}-1} g_{p}^{0(k)}(1) B_{j p}^{1 *} \\
& { }^{+} g_{k_{j}}^{1(k)}(1) B_{j}^{2 *}+\sum_{p=0}^{k_{j}-1} g_{p}^{1(k)}(1) B_{j p}^{2 *}=\check{\psi}_{j}^{(k)}(1) . \\
& k_{i}+l \leq 2 m-1, \quad k_{j}+k \leq 2 m-1 .
\end{aligned}
$$

If the system (4.18), (4.19) is solvable with respect to $f$ and $g$, then one can construct $P(x, y)=\sum_{s=1}^{4} h_{s}(x, y) P_{s}(x, y)$, where $P_{s}(x, y)$ are polynomials of order $2 m-1$, and $h_{s}(x, y)$ are cut-off functions mentioned above [1], which take the corresponding given values together with their derivatives at the angular points. Consequently, for $H_{1}-P$ we obtain a boundary value problem in which the right sides of the boundary conditions satisfy (4.1).

First we prove the solvability of (4.18), (4.19) when the boundary conditions are without low order terms. Let us express $g$ in terms of $f$ using (4.18) and substitute it into (4.19):

$$
\begin{gathered}
\left(\begin{array}{cc}
f_{k}^{0\left(k_{j}\right)}(0) & f_{k}^{0\left(k_{j}\right)}(1) \\
f_{k}^{1\left(k_{j}\right)}(0) & f_{k}^{1\left(k_{j}\right)}(1)
\end{array}\right)\left(\begin{array}{c}
B_{j}^{1 *} \\
B_{j}^{2 *}
\end{array}\right)=\left(\begin{array}{c}
\check{\psi}_{j}^{(k)}(0) \\
\check{\psi}_{j}^{(k)}(1)
\end{array}\right), \\
\left(\begin{array}{ll}
B_{i}^{1} & B_{i}^{2}
\end{array}\right)\left(\begin{array}{cc}
f_{k_{i}}^{0(l)}(0) & f_{k_{i}}^{0(l)}(1) \\
f_{k_{i}}^{1(l)}(0) & f_{k_{i}}^{1(l)}(1)
\end{array}\right)=\left(\begin{array}{lll}
\check{\varphi}_{i}^{(l)}(0) & \left.\check{\varphi}_{i}^{(l)}(1)\right)
\end{array}\right.
\end{gathered}
$$

Set

$$
\begin{aligned}
\left(\begin{array}{cc}
f_{q}^{0(r)}(0) & f_{q}^{0(r)}(1) \\
f_{q}^{1(r)}(0) & f_{q}^{1(r)}(1)
\end{array}\right) & =X_{q}^{r}, \quad\left(\begin{array}{c}
B_{j}^{1 *} \\
B_{j}^{2 *}
\end{array}\right)=B_{j}^{*}, \quad\left(\begin{array}{ll}
B_{i}^{1} & B_{i}^{2}
\end{array}\right)=B_{i}, \\
\left(\begin{array}{c}
\check{\psi}_{j}^{(k)}(0) \\
\check{\psi}_{j}^{(k)}(1)
\end{array}\right) & =\Psi_{j k}, \quad\left(\check{\varphi}_{i}^{(l)}(0) \quad \check{\varphi}_{i}^{(l)}(1)\right)=\Phi_{i l} .
\end{aligned}
$$

Then we can represent (4.20) as

$$
\text { 1. } X_{k}^{k_{j}} B_{j}^{*}=\Psi_{j k}, \quad \text { 2. } B_{i} X_{k_{i}}^{l}=\Phi_{i l} .
$$

The compatibility conditions (3.1) appear in the following form:

$$
B_{i} \Psi_{j k_{i}}=\Phi_{i k_{j}} B_{j}^{*}, \quad k_{i}+k_{j} \leq 2 m-1 .
$$

It can be easily seen that (4.21) decomposes into subsystems with respect 
to some $X_{q}^{r}$. Each of these subsystems can consist of one, two, three or four matrix equations depending on which boundary conditions are present in (3.7), (3.8). Note that these subsystems may consist of no more than two matrix equations of type 1 and no more than two matrix equations of type 2. Otherwise conditions (4.18) would connect the values of the functions $f_{k}^{i(l)}(y), k=0, \ldots, 2 m-1, l=0, \ldots, 2 m-1-k, i=0,1$ at the points $y=0$, $y=1$, which is impossible.

Let us now show that these subsystems are solvable in all cases mentioned above. Suppose we have one matrix equation with respect to $X_{q}^{r}$, for example of type 2 . Then

$$
B_{q} X_{q}^{r}=\Phi_{q r} .
$$

This equation is always solvable, because the rank of $B_{q}$ is $n$, due to the fact that the boundary conditions are regular.

Suppose we have two matrix equations of type 2 with respect to $X_{q}^{r}$,

$$
\left(\begin{array}{c}
B_{q} \\
B_{q+1}
\end{array}\right) X_{q}^{r}=\left(\begin{array}{c}
\Phi_{q r} \\
\Phi_{q+1, r}
\end{array}\right)
$$

This system is again solvable, because $\operatorname{det}\left(\begin{array}{c}B_{q} \\ B_{q+1}\end{array}\right)$ is not zero due to the fact that the boundary conditions (3.7), (3.8) are regular. Let us again consider system (4.21). Let $k=k_{i}, l=k_{j}$. Then (4.21) is transformed into

$$
\text { (a) } X_{k}^{l} B_{j}^{*}=\Psi_{j k}, \quad \text { (b) } B_{i} X_{k}^{l}=\Phi_{i l} \text {. }
$$

If (4.23) consists of four matrix equations, then again from the regularity of the boundary conditions we obtain

$$
X_{k}^{l}=\left(\begin{array}{ll}
\Psi_{j k} & \Psi_{j, k+1}
\end{array}\right)\left(\begin{array}{ll}
B_{j}^{*} & B_{j+1}^{*}
\end{array}\right)^{-1}=\left(\begin{array}{c}
B_{i} \\
B_{i+1}
\end{array}\right)^{-1}\left(\begin{array}{c}
\Phi_{i l} \\
\Phi_{i+1, l}
\end{array}\right) .
$$

It follows directly that

$$
B_{i} \Psi_{j k}=\Phi_{i l} B_{j}^{*}, \quad B_{i+1} \Psi_{j+1, k}=\Phi_{i+1, l} B_{j+1}^{*},
$$

and $k=k_{i}, l=k_{j}$, so (4.25) is one of the compatibility conditions (4.22). They are assumed to hold, so (4.23) has a solution, which is given by one of the formulas in (4.24).

Let (4.23) consist of one matrix equation of each type (the case when (4.23) consists of two matrix equations of one type and one matrix equation of another type is treated similarly). The rank of $B_{i}$ is $n$, so we can solve the second equation in (4.23) with respect to $X_{k}^{l}$ and write it in the following form:

$$
X_{k}^{l}=B_{i}^{+} \Phi_{i l},
$$

where $B_{i}^{+}$is some right-inverse operator to $B_{i}$, i.e., $B_{i} B_{i}^{+}=E$, not uniquely 
defined. Represent it in the following way:

$$
B_{i}^{+} \Phi_{i l}=C_{i} \Phi_{i l}+Y_{i},
$$

where $C_{i}$ is a linear operator which solves the corresponding nonhomogeneous problem and $Y_{i}$ is a solution of the homogeneous one. Select $B_{i}^{+}$in such a way that $X_{k}^{l}$, defined from the second matrix equation in (4.23), is also a solution of the first one. Then

$$
X_{k}^{l} B_{j}^{*}=B_{i}^{+} \Phi_{i l} B_{j}^{*}=\Psi_{j k} .
$$

Since the compatibility conditions $B_{i} \Psi_{j k}=\Phi_{i l} B_{j}^{*}$ hold, for solvability of (4.27) we must select $B_{i}^{+}$in such a way that

$$
C_{i} B_{i} \Psi_{j k}+Y_{i} B_{j}^{*}=\Psi_{j k} .
$$

Notice that $B_{i}$ acts in $\mathbb{C}_{2 n \times n}$. Decompose $\mathbb{C}_{2 n \times n}$ into the direct sum of the defective subspace and its orthogonal complement. Represent $\Psi_{j k}$ in the form

$$
\Psi_{j k}=\Psi_{j k}^{1}+\Psi_{j k}^{2}
$$

where $B_{i} \Psi_{j k}^{2}=0$ and $\Psi_{j k}^{1}$ belongs to the orthogonal complement. Let $E_{s}$, $s=1, \ldots, n$, be a basis in the orthogonal complement and $G_{s}, s=1, \ldots, n$, be a basis in the defective subspace. Let

$$
C_{i} B_{i} E_{s}=E_{s}, \quad s=1, \ldots, n, \quad Y_{i} B_{j}^{*}=\Psi_{j k}^{2} .
$$

From the regularity of the boundary conditions (3.7), (3.8) it follows that (4.29) is solvable with respect to $C_{i}$ and $Y_{i}$. The theory of linear algebraic systems [7] shows that among all solutions of (4.29) we can select $Y_{i}$ that can be represented in the following form:

$$
Y_{i}=\Psi_{j k}^{2} Y_{i}^{1}
$$

where $Y_{i}^{1}$ is a matrix of appropriate dimension. Notice that $B_{i} Y_{i}=0$. It is not difficult to verify that defined in such a way, the operator $B_{i}^{+}$satisfies (4.28). We can show that $B_{i}^{+} \Phi_{i l}$ is a solution of $B_{i} X_{k}^{l}=\Phi_{i l}$.

Represent $\Phi_{i l}$ in the following form:

$$
\Phi_{i l}=\left(\sum_{p=1}^{n} \alpha_{p} Q_{p} \quad \sum_{p=1}^{n} \beta_{p} Q_{p}\right),
$$

where $Q_{p}=B_{i} E_{p}, p=1, \ldots, n$. Consequently, by (4.29),

$$
\begin{aligned}
B_{i}^{+} \Phi_{i l} & =C_{i} \Phi_{i l}+Y_{i}=C_{i}\left(\sum_{p=1}^{n} \alpha_{p} Q_{p} \sum_{p=1}^{n} \beta_{p} Q_{p}\right)+Y_{i} \\
& =\left(\sum_{p=1}^{n} \alpha_{p} E_{i} \sum_{p=1}^{n} \beta_{p} E_{i}\right)+Y_{i} .
\end{aligned}
$$


Finally we obtain

$$
\begin{aligned}
B_{i} B_{i}^{+} \Phi_{i l} & =B_{i}\left(C_{i} \Phi_{i l}+Y_{i}\right) \\
& =B_{i}\left(\sum_{p=1}^{n} \alpha_{p} C_{i} Q_{p} \sum_{p=1}^{n} \beta_{p} C_{i} Q_{p}\right)+B_{i} Y_{i} \\
& =B_{i}\left(\sum_{p=1}^{n} \alpha_{p} E_{i} \sum_{p=1}^{n} \beta_{p} E_{i}\right)+B_{i} Y_{i} \\
& =\left(\sum_{p=1}^{n} \alpha_{p} Q_{p} \sum_{p=1}^{n} \beta_{p} Q_{p}\right)=\Phi_{i l}
\end{aligned}
$$

since $B_{i} Y_{i}=0$.

Suppose now that low order terms of the boundary conditions are present. In that case the compatibility conditions appear in the following form:

$$
\Phi_{i k_{j}} B_{j}^{*}+\sum_{p=0}^{k_{j}-1} \Phi_{i p} B_{j p}^{*}=B_{i} \Psi_{i k_{j}}+\sum_{p=0}^{k_{i}-1} B_{i p} \Psi_{j p}, \quad k_{i}+k_{j} \leq 2 m-1 .
$$

After replacing $g$ by $f$ the systems (a) and (b) take the form

$$
\begin{aligned}
& \text { (a) } X_{k}^{k_{j}} B_{j}^{*}+\sum_{q=0}^{k_{j}-1} X_{k}^{q} B_{j q}^{*}=\Psi_{j k}, \quad k+k_{j} \leq 2 m-1, \\
& \text { (b) } B_{i} X_{k_{i}}^{l}+\sum_{p=0}^{k_{i}-1} B_{i p} X_{p}^{l}=\Phi_{i l}, \quad k_{i}+l \leq 2 m-1 .
\end{aligned}
$$

Consider (b) for each $l=0, \ldots, 2 m-k_{i}-1$ to obtain $X_{0}^{l}, X_{1}^{l}, \ldots, X_{k_{i}}^{l}$. We can do this as the boundary conditions are regular and (b) has a diagonal form for each $l$. Let us prove that a suitable solution of (b) is also a solution of (a). Let $k_{i}$ and $k_{j}$ take values in $\left[\tau_{0}, \tau_{1}\right]$, where $0 \leq \tau_{0}, \tau_{1} \leq 2 m-1$. Then $0 \leq k, l \leq \tau_{2}=2 m-1-\tau_{0}$. If $\tau_{0}+\tau_{1} \leq 2 m-1$ then $\tau_{2} \geq \tau_{1}$ and the unknown terms $X_{k}^{\mu}, k>\tau_{1}, k+\mu \leq 2 m-1$, are obtained directly from (a). Note that if the boundary condition of order $k_{\nu}$, where $\tau_{0} \leq k_{\nu} \leq \tau_{1}$, is absent in (3.7) then we obtain $X_{k_{\nu}}^{\mu}$ for $\mu+k_{\nu} \leq 2 m-1$ again directly from (a), substitute them in (b) and obtain the corresponding $X_{k_{i}}^{l}$ for $k_{i}+l \leq 2 m-1$ from system (b). If $\tau_{0}+\tau_{1}>2 m-1$ then $\tau_{1}>\tau_{2}$. In that case $X_{k}^{\mu}$ for $\mu+k \leq 2 m-1$ and for $\mu>\tau_{2}$ are obtained directly from (a). Moreover if the boundary condition of order $k_{\nu}$ is absent in (3.7) then we use the same considerations as in the case $\tau_{0}+\tau_{1} \leq 2 m-1$. When $k_{\nu}>\tau_{2}$ we choose $X_{k_{\nu}}^{\mu}$ for $k_{\nu}+\mu \leq 2 m-1$ arbitrarily. Substitute $X_{k_{i}}^{l}, k_{i}+l \leq 2 m-1$, from (b) 
into (a), regarding that

$$
X_{k_{i}}^{l}=C_{k_{i}}\left(\Phi_{i l}-\sum_{p=0}^{k_{i}-1} B_{i p} X_{p}^{l}\right)+Y_{k_{i} l}
$$

where $B_{i} Y_{k_{i} l}=0, l=0, \ldots, \tau_{2}$. Using the corresponding compatibility conditions we see that $X_{k_{i}}^{l}, k_{i}+l \leq 2 m-1$, from (b) solve system (a) if and only if

$$
C_{k_{i}} B_{i} \Psi_{j k_{i}}+Y_{k_{i} k_{j}} B_{j}^{*}+\sum_{q=0}^{k_{j}} Y_{k_{i} q} B_{j q}^{*}=\Psi_{j k_{i}}, \quad k_{i}+k_{j} \leq 2 m-1 .
$$

We have already constructed such operators when proving the theorem in the case when the boundary conditions do not contain low order terms.

Note that if $k_{i}+k_{j}>2 m-1$ for all $i, j$ then every equation of system (a) contains terms which cannot be obtained from (b) and therefore it is possible to solve system (a)-(b) without compatibility conditions.

Finally, the values of the traces of $V(x, y)$ and their derivatives at the vertices of the square satisfy the conditions (4.18), (4.19) if and only if the compatibility conditions (3.1) hold. Moreover, if $k_{i}+k_{j}>2 m-1$ for all $i, j$, then $k_{l}, k_{s}<k_{i}, k_{j}$ and the system (4.18), (4.19) is solvable without the compatibility conditions (which are not required in this case). Therefore $V(x, y)=\widetilde{V}(x, y)+P(x, y)$ satisfies the boundary conditions $(3.7),(3.8)$ and has the required smoothness. The theorem is proved.

Thus $Z(x, y)=H_{1}(x, y)-V(x, y)$ is a solution of the boundary value problem

$$
\begin{aligned}
& \mathbf{A}_{1} Z(x, y)+\left[\mathbf{A}_{2} Z^{T}(x, y)\right]^{T}=F^{*}(x, y), \quad(x, y) \in \Omega, \\
& \mathbf{B}_{i 1} Z(x, y)+\mathbf{B}_{i 2} Z(x, y)=0, \quad i=1, \ldots, 2 m, \\
& \mathbf{C}_{j 1} Z(x, y)+\mathbf{C}_{j 2} Z(x, y)=0, \quad j=1, \ldots, 2 m,
\end{aligned}
$$

where $F^{*}(x, y)=\check{F}(x, y)-\left(\mathbf{A}_{1} V(x, y)+\left[\mathbf{A}_{2} V^{T}(x, y)\right]^{T}\right)$.

5. Solution of the homogeneous boundary value problem. Consider problem (4.31). Let $Z(x, y)$ and $F^{*}(x, y)$ be functions with values in $\mathbb{C}_{n^{2}}$. Then problem (4.31) can be represented in the following way:

$$
\begin{aligned}
& \mathbf{A}_{x} Z(x, y)+\mathbf{A}_{y} Z(x, y)=F(x, y) \\
& \left(B_{i}^{1} \otimes \mathbf{I}\right) Z_{x}^{\left(k_{i}\right)}(0, y)+\sum_{p=0}^{k_{i}-1}\left(B_{i p}^{1} \otimes \mathbf{I}\right) Z_{x}^{(p)}(0, y) \\
& +\left(B_{i}^{2} \otimes \mathbf{I}\right) Z_{x}^{\left(k_{i}\right)}(1, y)+\sum_{p=0}^{k_{i}-1}\left(B_{i p}^{2} \otimes \mathbf{I}\right) Z_{x}^{(p)}(1, y)=0, \quad i=1, \ldots, 2 m,
\end{aligned}
$$


$\begin{aligned} & (5.1) \\ & \text { [cont.] }\end{aligned}\left(\mathbf{I} \otimes \bar{B}_{j}^{1}\right) Z_{y}^{\left(k_{j}\right)}(x, 0)+\sum_{p=0}^{k_{j}-1}\left(\mathbf{I} \otimes \bar{B}_{j p}^{1}\right) Z_{y}^{(p)}(x, 0)$

$$
+\left(\mathbf{I} \otimes \bar{B}_{j}^{2}\right) Z_{y}^{\left(k_{j}\right)}(x, 1)+\sum_{p=0}^{k_{j}-1}\left(\mathbf{I} \otimes \bar{B}_{j p}^{2}\right) Z_{y}^{(p)}(x, 1)=0, \quad j=1, \ldots, 2 m,
$$

where

$$
\begin{aligned}
\mathbf{A}_{x} Z(x, y)= & Z_{x}^{(2 m)}(x, y)+(-1)^{2 m-1}\left(\left(P_{1}^{*} \otimes \mathbf{E}\right) Z(x, y)\right)^{(2 m-1)} \\
& +(-1)^{2 m-2}\left(\left(P_{2}^{*} \otimes \mathbf{E}\right) Z(x, y)\right)^{(2 m-2)}+\ldots+\left(P_{2 m}^{*} \otimes \mathbf{E}\right) Z(x, y), \\
\mathbf{A}_{y} Z(x, y)= & Z_{y}^{(2 m)}(x, y)+(-1)^{2 m-1}\left(\left(\mathbf{E} \otimes P_{1}^{T}\right) Z(x, y)\right)^{2 m-1} \\
& +(-1)^{2 m-2}\left(\left(\mathbf{E} \otimes P_{2}^{T}\right) Z(x, y)\right)+\ldots+\left(\mathbf{E} \otimes P_{2 m}^{T}\right) Z(x, y),
\end{aligned}
$$

and the symbol $\otimes$ means direct product. For simplicity we omit the star on $F(x, y)$. Let us prove that the solution of (5.1) is given by the contour integral (see [8])

$$
Z(x, y)=-\frac{1}{2 \pi i} \int_{\Gamma}\left(\mathbf{A}_{x}-\lambda \mathbf{I}\right)^{-1}\left(\mathbf{A}_{y}+\lambda \mathbf{I}\right)^{-1} F d \lambda .
$$

Here

$$
\left(\mathbf{A}_{x}-\lambda \mathbf{I}\right)^{-1} F=\int_{0}^{1} G(x, \xi, \lambda) F(\xi, \eta) d \xi
$$

where $G(x, \xi, \lambda)$ is the Green function of the operator $\mathbf{A}_{x}-\lambda \mathbf{I}$, and

$$
\left(\mathbf{A}_{y}+\lambda \mathbf{I}\right)^{-1} F=\int_{0}^{1} J(y, \eta, \lambda) F(\xi, \eta) d \eta
$$

where $J(y, \eta, \lambda)$ is the Green function of $\mathbf{A}_{y}+\lambda \mathbf{I}$. Moreover, $\Gamma$ is a composite contour, which consists of contours $\Gamma_{0}, \Gamma_{1}, \Gamma_{2}$ to be defined below (see [1]). From $1^{\mathrm{o}}-2^{\mathrm{o}}$ it follows that the spectrum of $\mathbf{A}_{x}$ lies in the sector $\mid \arg (\lambda-$ $\left.\gamma_{1}\right) \mid \leq \theta_{1}$ and the spectrum of $-\mathbf{A}_{y}$ lies in the sector $\left|\arg \left(\lambda-\gamma_{2}\right)\right| \leq \theta_{2}$, where $\gamma_{1}, \gamma_{2} \geq 0, \pi / 2<\theta_{1}<\pi, 0<\theta_{2}<\pi / 2$. We encircle the part of the spectrum of $\mathbf{A}_{x}$ in the right half-plane by some contour $\Gamma_{1}$ so that the points of the spectrum of $-\mathbf{A}_{y}$ lie outside the contour. We proceed similarly with the part of the spectrum of $-\mathbf{A}_{y}$ in the left half-plane, using a contour $\Gamma_{2}$. It is obvious that these contours may be constructed, because the spectra of $\mathbf{A}_{x}$ and $-\mathbf{A}_{y}$ are disjoint. For $\Gamma_{0}$ we take the imaginary axis, which does not intersect the two spectra. The integral over $\Gamma_{0}$ is understood as a principal value. The convergence of (5.2) and its derivatives over $\Gamma_{1}$ and $\Gamma_{2}$ is easily determined.

Below we are interested in the behavior of the contour integral (5.2) and its derivatives in $L_{2}\left(\Omega, \mathbb{C}_{n^{2}}\right)$. It is clear that the convergence of (5.2) is 
determined by the convergence of the integral over $\Gamma_{0}$. Moreover, it is not difficult to show from the properties of the direct product that $\mathbf{A}_{x}, \mathbf{A}_{y}$ and their resolvents commute.

Denote by $\varrho$ that value of $\sqrt[2 m]{\lambda}$ which has the smallest positive real part. Write the asymptotic expansions of the Green function as $|\lambda| \rightarrow \infty$ [13]:

$$
\begin{aligned}
& G(x, \xi, \lambda)=\frac{C}{\varrho^{2 m-1}} \exp \varrho(x-\xi) \mathbf{I}+O\left(\varrho^{2 m}\right), \quad x<\xi, \\
& G(x, \xi, \lambda)=\frac{C}{\varrho^{2 m-1}} \exp \varrho(\xi-x) \mathbf{I}+O\left(\varrho^{2 m}\right), \quad \xi<x,
\end{aligned}
$$

where $O\left(\varrho^{2 m}\right)$ means a vector of the type $D(x, \xi, \lambda) / \varrho^{2 m}$, where $D(x, \xi, \lambda)$ is a function with values in $\mathbb{C}_{n^{2}}$ whose entries all satisfy

$$
\left|D_{i}(x, \xi, \lambda)\right| \leq C,
$$

where $C$ is some constant. We can have a similar representation for $J(y, \eta, \lambda)$. Recall that, without loss of generality, we can suppose that for all $|\lambda|$,

$$
\left\|\left(\mathbf{A}_{x}-\lambda \mathbf{I}\right)^{-1}\right\| \leq \frac{C}{1+|\lambda|}, \quad\left\|\left(\mathbf{A}_{y}+\lambda \mathbf{I}\right)^{-1}\right\| \leq \frac{C}{1+|\lambda|} .
$$

First, let us prove that differentiation and integration commute. Consider the integral

$$
\int_{\Gamma}\left(\mathbf{A}_{y}+\lambda \mathbf{I}\right)^{-1} \mathbf{A}_{x}\left(\mathbf{A}_{x}-\lambda \mathbf{I}\right)^{-1} F d \lambda
$$

and let us investigate its convergence in $L_{2}\left(\Omega, \mathbb{C}_{n^{2}}\right)$.

Lemma 2. Suppose that $F \in W_{2}^{\varepsilon}\left(\Omega, \mathbb{C}_{n^{2}}\right)$. Then the integral (5.5) converges in $L_{2}\left(\Omega, \mathbb{C}_{n^{2}}\right)$.

Proof. Consider the identity

$$
\mathbf{A}_{x}\left(\mathbf{A}_{x}-\lambda \mathbf{I}\right)^{-1} F=F+\lambda\left(\mathbf{A}_{x}-\lambda \mathbf{I}\right)^{-1} F .
$$

Substitute (5.6) into (5.5) to get

$$
\begin{aligned}
\int_{\Gamma}\left(\mathbf{A}_{y}+\lambda \mathbf{I}\right)^{-1} \mathbf{A}_{x}\left(\mathbf{A}_{x}-\lambda \mathbf{I}\right)^{-1} F d \lambda \\
=\int_{\Gamma}\left(\mathbf{A}_{y}+\lambda \mathbf{I}\right)^{-1} F d \lambda+\int_{\Gamma}\left(\mathbf{A}_{y}+\lambda \mathbf{I}\right)^{-1} \lambda\left(\mathbf{A}_{x}-\lambda \mathbf{I}\right)^{-1} F d \lambda .
\end{aligned}
$$

Using Lemma 1 from [1], identity (5.6) and the Minkowski inequality we have

$$
\begin{aligned}
\left\|\int_{\Gamma}\left(\mathbf{A}_{y}+\lambda \mathbf{I}\right)^{-1} \mathbf{A}_{x}\left(\mathbf{A}_{x}-\lambda \mathbf{I}\right)^{-1} F d \lambda\right\| \\
\leq\left\|\int_{\Gamma}\left(\mathbf{A}_{y}+\lambda \mathbf{I}\right)^{-1} \lambda\left(\mathbf{A}_{x}-\lambda \mathbf{I}\right)^{-1} F d \lambda\right\|+C\|F\|,
\end{aligned}
$$


where $\|\cdot\|=\|\cdot\|_{L_{2}\left(\Omega, \mathbb{C}_{n^{2}}\right)}$. It remains to show that the norm of the integral on the right side of (5.8) is finite. It is clear that the difficulties may arise only when estimating the main term in the asymptotic formulas (5.3), (5.4). Denote it by $G_{0}(x, \xi, \lambda)$ and substitute it into the integral in question. Then add and subtract $F(x, \eta)$ in the resulting integral. By assumption, $F \in W_{2}^{\varepsilon}\left(\Omega, \mathbb{C}_{n^{2}}\right)$. With the help of integral representations of $W_{2}^{\varepsilon}\left(\Omega, \mathbb{C}_{n^{2}}\right)$ functions [14] one can show that $F \in W_{2}^{\varepsilon}\left(x, \mathbb{C}_{n^{2}}\right)$ for almost all $y$ and analogously $F \in W_{2}^{\varepsilon}\left(y, \mathbb{C}_{n^{2}}\right)$ for almost all $x$. Therefore

$$
\frac{F(\xi, \eta)-F(x, \eta)}{(|\xi-x|)^{\varepsilon+1 / 2}} \in L_{2}\left((0,1) \times(0,1), \mathbb{C}_{n^{2}}\right)
$$

for almost all $\eta$. Taking into account that $\mathbf{A}_{y}$ satisfies $1^{\circ}$, applying the Hölder inequality and again using Lemma 1 from [1], we obtain

$$
\begin{aligned}
\| \int_{\Gamma}\left(\mathbf{A}_{y}\right. & +\lambda \mathbf{I})^{-1} \lambda \int_{0}^{1} G_{0}(x, \xi, \lambda) F(\xi, \eta) d \xi d \lambda \| \\
\leq & C\|F\|+\int_{\Gamma} \frac{C}{|\varrho|^{2 m-1}}\left(\int _ { 0 } ^ { 1 } \left[\int_{-\infty}^{x}|\exp [2 \varrho(\xi-x)]| \cdot|\xi-x|^{2 \varepsilon+1} d \xi\right.\right. \\
& \left.\left.\times \int_{0}^{x} \frac{|F(\xi, \eta)-F(x, \eta)|^{2}}{|\xi-x|^{2 \varepsilon+1}} d \xi\right] d x\right)^{1 / 2}|d \lambda| \\
& +\int_{\Gamma} \frac{C}{|\varrho|^{2 m-1}}\left(\int _ { 0 } ^ { 1 } \left[\int_{x}^{\infty}|\exp [2 \varrho(x-\xi)]| \cdot|x-\xi|^{2 \varepsilon+1} d \xi\right.\right. \\
& \left.\left.\times \int_{x}^{1} \frac{|F(\xi, \eta)-F(x, \eta)|^{2}}{|\xi-x|^{2 \varepsilon+1}} d \xi\right] d x\right)^{1 / 2}|d \lambda| .
\end{aligned}
$$

Calculating the integrals in square brackets one obtains

$$
\begin{aligned}
\left\|\int_{\Gamma}\left(\mathbf{A}_{y}+\lambda \mathbf{I}\right)^{-1} \lambda \int_{0}^{1} G_{0}(x, \xi, \lambda) F(\xi, \eta) d \xi d \lambda\right\| \\
\quad \leq \int_{\Gamma} \frac{C}{1+|\lambda|^{1+\varepsilon /(2 m)}}|d \lambda| \cdot\|F\|_{W_{2}^{\varepsilon}\left(\Omega, \mathbb{C}_{n^{2}}\right)} .
\end{aligned}
$$

Obviously the integral on the right side of (5.10) absolutely converges when $\varepsilon>0$. From (5.8) and (5.10) it follows that (5.5) converges in $L_{2}\left(\Omega, \mathbb{C}_{n^{2}}\right)$. Analogously one can show that the integral

$$
\int_{\Gamma} \mathbf{A}_{y}\left(\mathbf{A}_{y}+\lambda \mathbf{I}\right)^{-1}\left(\mathbf{A}_{x}-\lambda \mathbf{I}\right)^{-1} F d \lambda
$$

converges in $L_{2}\left(\Omega, \mathbb{C}_{n^{2}}\right)$ when $F \in W_{2}^{\varepsilon}\left(\Omega, \mathbb{C}_{n^{2}}\right)$. The lemma is proved. 
Let us now show that the operator of differentiation can be inserted under the integral sign.

Lemma 3. If the resolvents $R\left(\mathbf{A}_{x}, \lambda\right), R\left(\mathbf{A}_{y}, \lambda\right)$ satisfy $1^{\circ}$ with appropriate $\theta$ and $\gamma$, then

$$
\mathbf{A}_{x} \int_{\Gamma}\left(\mathbf{A}_{y}+\lambda \mathbf{I}\right)^{-1}\left(\mathbf{A}_{x}-\lambda \mathbf{I}\right)^{-1} F d \lambda=\int_{\Gamma}\left(\mathbf{A}_{y}+\lambda \mathbf{I}\right)^{-1} \mathbf{A}_{x}\left(\mathbf{A}_{x}-\lambda \mathbf{I}\right)^{-1} F d \lambda .
$$

Proof. Let

$$
R(x, y, \lambda)=R\left(\mathbf{A}_{y}, \lambda\right) R\left(\mathbf{A}_{x}, \lambda\right) F(x, y) .
$$

We have to prove that

$$
\mathbf{A}_{x} \int_{\Gamma} R(x, y, \lambda) d \lambda=\int_{\Gamma} \mathbf{A}_{x} R(x, y, \lambda) d \lambda .
$$

The validity of this equality for the integrals over $\Gamma_{1}$ and $\Gamma_{2}$ follows from their boundedness and the properties of the resolvents $R\left(\mathbf{A}_{x}, \lambda\right), R\left(\mathbf{A}_{y}, \lambda\right)$. Consider a sequence of bounded contours $\Gamma_{0}^{n}$ such that $\lim _{n \rightarrow \infty} \Gamma_{0}^{n}=\Gamma_{0}$. Using the considerations from the beginning of the section, for all $n$ we have

$$
\mathbf{A}_{x} \int_{\Gamma_{0}^{n}} R(x, y, \lambda) d \lambda=\int_{\Gamma_{0}^{n}} \mathbf{A}_{x} R(x, y, \lambda) d \lambda .
$$

Using Lemma 2 and the fact that $\mathbf{A}_{x}$ is a closed operator we obtain

$$
\mathbf{A}_{x} \int_{\Gamma_{0}} R(x, y, \lambda) d \lambda=\int_{\Gamma_{0}} \mathbf{A}_{x} R(x, y, \lambda) d \lambda .
$$

Analogously one can prove that

$$
\mathbf{A}_{y} \int_{\Gamma_{0}} R(x, y, \lambda) d \lambda=\int_{\Gamma_{0}} \mathbf{A}_{y} R(x, y, \lambda) d \lambda .
$$

The lemma is proved.

Let us show that (5.2) gives a solution of (5.1).

Lemma 4. Suppose that $F \in W_{2}^{\varepsilon}\left(\Omega, \mathbb{C}_{n^{2}}\right)$. Then (5.2) gives a solution of (5.1).

Substituting (5.2) into (5.1), using Lemma 1 from [1] and the identities

$$
\begin{aligned}
& \mathbf{A}_{x}\left(\mathbf{A}_{x}-\lambda \mathbf{I}\right)^{-1} F=F+\lambda\left(\mathbf{A}_{x}-\lambda \mathbf{I}\right)^{-1} F, \\
& \mathbf{A}_{y}\left(\mathbf{A}_{y}+\lambda \mathbf{I}\right)^{-1} F=F-\lambda\left(\mathbf{A}_{y}+\lambda \mathbf{I}\right)^{-1} F,
\end{aligned}
$$

we obtain

$$
\mathbf{A}_{x} Z(x, y)+\mathbf{A}_{y} Z(x, y)=F(x, y) .
$$

The lemma is proved.

Consequently, if $F \in W_{2}^{\varepsilon}\left(\Omega, \mathbb{C}_{n^{2}}\right)$ then (5.2) gives the solution of (5.1), where $Z \in W_{2}^{2}\left(\Omega, \mathbb{C}_{n^{2}}\right)$. From the previous section one can see that $F(x, y)$ 
on the right side of the equation in (5.1) is in $W_{2}^{\varepsilon}\left(\Omega, \mathbb{C}_{n^{2}}\right)$. Therefore the kernel $U(x, y)$ of the integral operator $\mathbf{U}$ is represented as the sum

$$
U(x, y)=T(x, y)+v(x, y)+V(x, y)+Z(x, y),
$$

where all terms on the right side are regarded as functions with values in $\mathbb{C}_{n \times n}$.

Let us formulate the final result. We have proved the following

Theorem 3. Suppose that the spectra of $\mathbf{A}^{*}$ and $\mathbf{- A}$ are disjoint and the resolvents $R\left(\mathbf{A}^{*}, \lambda\right), R(-\mathbf{A}, \lambda)$ satisfy condition $1^{\circ}$. Then there exists a solution of the problem I-III and the kernel $U(x, y)$ of the integral operator

$$
\mathbf{U} u(x)=\int_{0}^{1} U(x, y) u(y) d y
$$

satisfies conditions 1-3.

So we have shown that (5.11) gives the unique solution of the Lyapunov equation

$$
\mathbf{A}^{*} \mathbf{U}+\mathbf{U A}=\mathbf{I}
$$

in the class of bounded operators.

\section{References}

[1] V. S. Belonosov, Instability indices of unbounded operators. I, in: Some Applications of Functional Analysis to Problems of Mathematical Physics, Proc. Sem. Sobolev., 1984, Part 2, Inst. Mat. Sibirsk. Otdel. Akad. Nauk SSSR, Novosibirsk, 1984, 25-51 (in Russian).

[2] -, Instability indices of unbounded operators. II, in: Some Applications of Functional Analysis to Problems of Mathematical Physics, Proc. Sem. Sobolev., Inst. Mat. Sibirsk. Otdel. Akad. Nauk SSSR, Novosibirsk, 1985, 5-33 (in Russian).

[3] - Instability indices of differential operators, Math. USSR-Sb. 57 (1987), 507-525.

[4] V. S. Belonosov, M. P. Vishnevskiǔ, T. I. Zelenyak and M. M. Lavrent'ev, Jr., On qualitative properties of solutions of parabolic equations, preprint No. 466, Computation Center, Siberian Branch Acad. Sci. USSR, Novosibirsk, 1983 (in Russian); Ref. Zh. Mat. 1984, 2585.

[5] V. Ya. Belov, On a formula of Morse in the critical case, Dokl. Akad. Nauk SSSR 274 (1984), 269-272 (in Russian); English transl. in Soviet Math. Dokl. 29 (1984).

[6] Yu. L. Daletskiı̌ and M. G. Kreĭn, Stability of Solutions of Differential Equations in Banach Spaces, Nauka, Moscow, 1970 (in Russian); English transl., Amer. Math. Soc., Providence, RI, 1974.

[7] F. R. Gantmakher, Theory of Matrices, Nauka, Moscow, 1967 (in Russian).

[8] P. Grisvard, Équations différentielles abstraites, Ann. Sci. École Norm. Sup. 2 (1969), 311-395.

[9] V. A. Kondrat'ev, Boundary value problems for elliptic equations in domains with conical points, Trudy Moskov. Mat. Obshch. 16 (1967), 209-292 (in Russian); English transl. in Trans. Moscow Math. Soc. 16 (1967). 
[10] S. G. Kreĭn, Linear Differential Operators in Banach Space, Nauka, Moscow, 1969 (in Russian).

[11] M. Morse, A generalization of Sturm separation and comparison theorems in $n$ space, Math. Ann. 103 (1930), 52-69.

[12] - Variational Analysis: Critical Extremals and Sturmian Extensions, Wiley, New York, 1973.

[13] M. A. Nămark, Linear Differential Operators, 2nd ed., Nauka, Moscow, 1969 (in Russian); English transl. of 1st ed., Parts I, II, Ungar, New York, 1967, 1968.

[14] S. M. Nikol'skiı̌, Approximation of Functions of Several Variables and Imbedding Theorems, Nauka, Moscow, 1977 (in Russian).

Institute of Applied and Computing Mathematics

FO.R.T.H. Heraklion, Crete 71110, Greece

E-mail: tersenov@sonar.iacm.forth.gr 